\title{
A Survey of Menopause Care Among US Women
}

\author{
Sandy Rose Truong ${ }^{1}$, Jennifer Wolff ${ }^{2}$, Teresa Keenan ${ }^{2}$, Victoria Gelfeld ${ }^{2}$, G. Oscar Anderson ${ }^{2}$, \\ Patricia David $^{2}$, Wen Shen ${ }^{1, *}$
}

${ }^{1}$ School of Medicine, Department of Gynecology and Obstetrics, Johns Hopkins University, Baltimore, United States

${ }^{2}$ American Association of Retired Persons (AARP), Washingto, United States

Email address:

wshen1@jhmi.edu (Wen Shen)

${ }^{*}$ Corresponding author

\section{To cite this article:}

Sandy Rose Truong, Jennifer Wolff, Teresa Keenan, Victoria Gelfeld, G. Oscar Anderson, Patricia David, Wen Shen. A Survey of Menopause Care Among US Women. Journal of Gynecology and Obstetrics. Vol. 7, No. 2, 2019, pp. 31-35. doi: 10.11648/j.jgo.20190702.11

Received: January 7, 2019; Accepted: February 25, 2019; Published: April 18, 2019

\begin{abstract}
Objective: Nearly one-third of American women are postmenopausal and at risk for menopausal symptoms such as hot flashes, mood disorders, and vulvovaginal atrophy. Primary healthcare providers in addition to gynecologists need to be ready to address these concerns. One area of controversy has been the role of menopausal hormone therapy (MHT) in the treatment of symptoms. This study sought to determine how often women seek care for menopause, their satisfaction with care, and their use of MHT. Methods: An online survey was administered to 1,509 women age 40-89. Descriptive statistics were performed. Results: $81 \%$ have experienced symptoms but only $50 \%$ of women have discussed menopause with a provider and $31 \%$ do not receive information about menopause from any sources. Among those who say they need treatment, only $15 \%$ have pursued but not received it. Of those who have discussed it with their provider, $25 \%$ with symptoms were not offered treatment and $11 \%$ said their provider was not sympathetic. However, $97 \%$ said that their provider was comfortable and $97 \%$ knowledgeable. Regarding treatment, $62 \%$ reported that providers discussed MHT, 36\% reported that providers recommended it, but only $6 \%$ of women are using it. Conclusions: It is encouraging that the vast majority of women who have pursued treatment are satisfied with their care. However, a quarter of women who sought treatment were not offered treatment and half of the women surveyed had never been counselled on menopause. Steps should be taken to encourage provider-initiated discussion of menopause.
\end{abstract}

Keywords: Quality of Care, Hormone Replacement Therapy, Menopausal Hormone Therapy, Menopause Care

\section{Introduction}

As life expectancy increases in the United States, a growing number of women is postmenopausal, now making up nearly one-third of the female population based on the United States census. [1] This group is at higher risk for postmenopausal health issues such as cardiovascular disease, cancer, and osteoporosis, which are often managed by primary healthcare providers. They are also at risk for vasomotor symptoms, mood disorders, and vulvovaginal atrophy that can drastically impact quality of life. Primary healthcare providers need to be ready to address these concerns, manage these symptoms, and prevent morbidity and mortality.

A series of surveys in North America and Europe from 2000 to 2012 have shown that healthcare professionals are the most frequent source of information for menopausal symptoms, but a large range, from 23 to $72 \%$, of women did not consult a healthcare professional for their symptoms. [26] Moreover, of those who did consult a provider, 65 to $85 \%$ of the women had to initiate the discussion. [2, 5] These studies suggest that menopausal symptoms have not been adequately addressed by healthcare professionals.

This is complicated by the controversial role of menopausal hormone therapy (MHT) for these symptoms. Following the Women's Health Initiative (WHI) study that suggested an increased risk of adverse outcomes such as heart disease, stroke, and breast cancer, the use of MHT declined significantly to reach an estimated low of $4.7 \%$ in 2010. [7-10] In the same series of surveys on menopausal symptoms, healthcare professionals offered MHT to approximately $20 \%$ of their patients and about 20 to $30 \%$ of 
women reported current MHT use. [3, 5, 11] However, further analyses of the WHI data and newer studies have shown no increased risk with possible protective cardiovascular effects of MHT in younger women. [12-15]

Because of the increased awareness of lack of menopause care from these studies and newer data demonstrating the safety of MHT in select populations, we conducted the survey to determine how often women discuss symptoms with providers, their satisfaction with their care and treatment recommendations, and their opinion of MHT.

\section{Methods}

A 25-question online survey was created with questions regarding demographics, menopausal status and symptoms, sources of information on menopause, rating of healthcare providers on menopause care, and opinions toward MHT. This study was exempt from institutional review board due to minimal to no risk to participants. This survey was administered in English to women living in the United States between age 40-89 in April 2018 from a non-probability panel comprising of the AARP's five thousand panelists and
Toluna's four million panelists until the quota of 1500 completed surveys with an oversample of Hispanics (goal of 425) was reached. Descriptive statistics were performed for primary analysis, stratified by menopausal status.

\section{Results}

There were a total of 1509 respondents. In our sample of women age $40-89,16 \%$ of women considered themselves premenopausal (asymptomatic with regular bleeding), 10\% perimenopausal (some symptoms with irregular bleeding), $10 \%$ menopausal (some symptoms with cessation of bleeding within last year), and 64\% postmenopausal (no symptoms with cessation of bleeding more than a year ago). In total, $81 \%$ have experienced menopause symptoms in the past or currently with the most common symptom being hot flashes (31\%). Among those who have menopausal symptoms, $8 \%$ say symptoms interfere with their lives "a great deal" and $1 \%$ describe symptoms as "completely debilitating." Tables 1 and 2 summarizes the demographics of the respondents by age and race for self-described menopausal status.

Table 1. Menopausal status by age (\%).

\begin{tabular}{lllll}
\hline- & Premenopausal* & Perimenopausal $\dagger$ & Menopausal\$ \\
\hline $40-49$ & 83 & 76 & 25 & \\
$50-59$ & 14 & 21 & 64 & 26 \\
$60-69$ & 1 & 1 & 8 & 35 \\
$70-79$ & 2 & 2 & 3 & 28 \\
$80-89$ & 0 & 0 & 0 & 7 \\
\hline
\end{tabular}

* No symptoms of menopause and menstruation patterns are as they have always been

$\uparrow$ Some symptoms of menopause and have seen changes in menstruation patterns

† Menstruation stopped within the past year, and have regular menopause symptoms

$\S$ Menstruation stopped more than a year ago and no longer experience any menopause symptoms

Table 2. Menopausal status by race (\%).

\begin{tabular}{|c|c|c|c|c|}
\hline-- & Premenopausal & Perimenopausal & Menopausal & Postmenopausal \\
\hline American Indian or Alaska Native & 5 & 1 & 5 & 2 \\
\hline Asian & 4 & 8 & 5 & 2 \\
\hline Black or African American & 14 & 16 & 17 & 10 \\
\hline Native Hawaiian or other Pacific Islander & 0 & 2 & 0 & 1 \\
\hline White & 69 & 71 & 66 & 85 \\
\hline Other & 9 & 5 & 10 & 3 \\
\hline
\end{tabular}

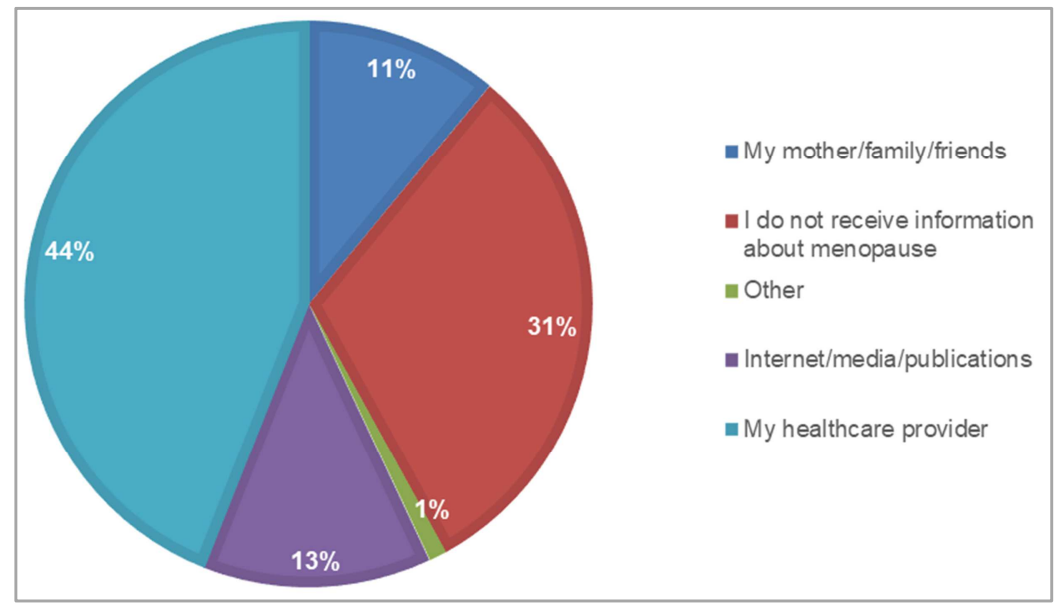

Figure 1. Respondents reported primary source of information about menopause. 
Figure 1 shows respondents' self-reported primary source of information about menopause, with the greatest number $(44 \%)$ receiving information from healthcare providers followed by $31 \%$ not receiving any information about menopause from any sources. For those who have consulted healthcare providers, over half sought care from primary care providers or general practitioners $(59 \%)$ and roughly the same from gynecologists/obstetricians (62\%) (Figure 2).

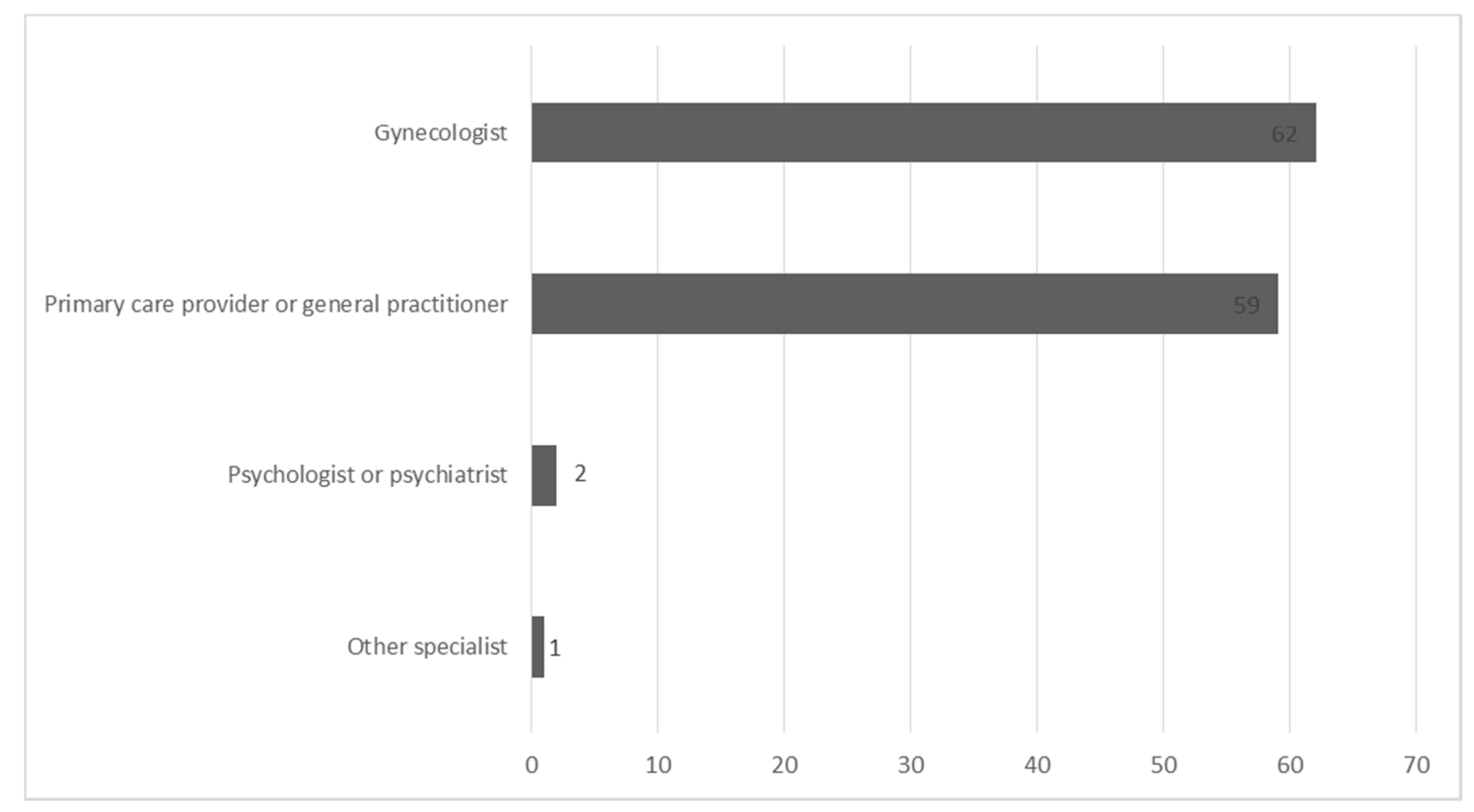

Figure 2. Types of healthcare providers with whom respondents have discussed menopause.

Overall, respondents rated their providers' knowledge on and comfort discussing menopause satisfactorily (Table 3). However, among women who report they are perimenopausal, menopausal, or postmenopausal and have discussed menopausal symptoms with a provider, they reported that only 57 to $67 \%$ of providers offered treatment for their symptoms,
51 to $66 \%$ had discussed MHT, and only 27 to $42 \%$ had recommended MHT. This is reflected in the low proportion of 7 to $17 \%$ of perimenopausal or menopausal women currently on MHT and the overwhelming proportion of women who feel they do not know enough about MHT to have formulated an opinion (34 to 47\%) as demonstrated in Table 4.

Table 3. Respondents' rating of care by provider (\%).

\begin{tabular}{|c|c|c|c|c|}
\hline-- & Premenopausal & Perimenopausal & Menopausal & Postmenopausal \\
\hline \multicolumn{5}{|c|}{ Did your provider seem comfortable discussing menopause? } \\
\hline Yes & 97 & 99 & 97 & 97 \\
\hline No & 3 & 1 & 3 & 3 \\
\hline \multicolumn{5}{|c|}{ Did your provider seem knowledgeable about menopause? } \\
\hline Yes & 98 & 97 & 95 & 97 \\
\hline No & 2 & 3 & 5 & 3 \\
\hline \multicolumn{5}{|c|}{ Was your provider sympathetic to your symptoms? } \\
\hline Yes & 61 & 90 & 85 & 80 \\
\hline No & 7 & 7 & 10 & 6 \\
\hline Not applicable & 32 & 3 & 4 & 14 \\
\hline \multicolumn{5}{|c|}{ Did your provider offer to treat your symptoms? } \\
\hline Yes & 24 & 59 & 67 & 57 \\
\hline No & 36 & 23 & 27 & 15 \\
\hline Not applicable & 40 & 18 & 5 & 28 \\
\hline \multicolumn{5}{|c|}{ Did your provider discuss menopausal hormone therapy? } \\
\hline Yes & $37-1$ & 51 & 64 & 66 \\
\hline No & 63 & 49 & 36 & 34 \\
\hline \multicolumn{5}{|c|}{ Did your provider recommend menopausal hormone therapy? } \\
\hline Yes & 25 & 27 & 42 & 36 \\
\hline No & 75 & 73 & 58 & 64 \\
\hline
\end{tabular}


Table 4. Respondents' attitude toward menopausal hormone therapy (\%).

\begin{tabular}{|c|c|c|c|c|}
\hline & Premenopausal & Perimenopausal & Menopausal & Postmenopausal \\
\hline \multicolumn{5}{|c|}{ Are you currently on menopausal hormone therapy for menopause? } \\
\hline Yes & 2 & 7 & 17 & 5 \\
\hline No & 98 & 93 & 83 & 95 \\
\hline \multicolumn{5}{|c|}{ What is your opinion of menopausal hormone therapy? } \\
\hline Very positive & 4 & 3 & 9 & 6 \\
\hline Somewhat positive & 6 & 9 & 10 & 9 \\
\hline Neutral & 26 & 28 & 18 & 21 \\
\hline Somewhat negative & 12 & 18 & 22 & 17 \\
\hline Very negative & 4 & 5 & 8 & 10 \\
\hline $\begin{array}{l}\text { I don't know enough about hormone } \\
\text { therapy to have an opinion }\end{array}$ & 47 & 38 & 34 & 36 \\
\hline
\end{tabular}

\section{Discussion}

A quarter of all women who sought treatment were not offered treatment and half of all the women surveyed had never been counselled on menopause health. Menopausal symptom screening and treatment counseling is complex and cannot be performed adequately in the time constraints of routine annual visits. We recommend routine screening for menopausal symptoms at annual visits and if positive, bringing the patient back for a more thorough counseling session on management of menopause symptoms including counseling on whether patients are candidates for MHT. In cases of patients with serious medical comorbidities, they should be referred to a specialized menopause practitioner for consultation of treatment for their menopause symptoms.

Despite newer research suggesting the safety of MHT, our study suggests that providers do not always include MHT in their counseling and even when they do, many women still do not feel they have been counseled adequately on MHT to have an opinion on it, suggesting that provider counseling may actually be inadequate. Among those whose providers discussed MHT, only a half to two-thirds of their providers actually recommended MHT, which may be one reason for the low proportion of women who are currently on MHT for their symptoms. The decision to prescribe MHT is a complicated one based on risk-stratification. Resources such as the Menopause Decision-Support Algorithm and MenoPro app from the North American Menopause Society have been developed to help aid this process and reflect the most current research. $[13,16]$ It remains to be seen how this will impact MHT use. There are also non-hormonal options for treatment of menopause symptoms, which have good evidence supporting efficacy, and should be discussed thoroughly with the patient to help her make the best decision for her health.

Although respondents were generally very satisfied with their menopause care and felt that their providers were knowledgeable, it is possible that many women do not receive any care because of providers' lack of comfort and knowledge in screening for and counseling effectively in menopause health. One study revealed that most obstetrics/gynecology residents felt they had limited knowledge of and needed to learn more about menopause medicine. [17] A dedicated menopause curriculum effectively improved knowledge base of residents to adequately manage menopause symptoms. [18] Similar steps can be taken in training programs for healthcare providers such as general practitioners and primary care physicians whom patients often consult for menopause symptoms.

There are several limitations to our study. This is a convenience sampling of women in the United States and thus subject to selection bias, possibly toward women with more severe menopausal symptoms or more willingness to discuss their symptoms. Moreover, the online panelists could reflect more highly educated or higher socioeconomic status, and are certainly not reflective of the racial demographics of the United States. Because the study was not conducted in the same manner and population as the previous series of surveys, the data cannot be directly compared to determine a trend. Another limitation of this study is the inability to discern whether respondents are not suitable candidates for MHT or whether providers are hesitant to recommend MHT even to suitable candidates. We were also unable to obtain information on whether alternative options to MHT were offered to respondents.

\section{Conclusions}

The results of this study suggest that there has not been any increase in care offered to women for menopause over the past decade. Given the progression of science and knowledge regarding the impact of menopause on women's health, this is a deficiency which needs to be addressed. Healthcare providers should routinely screen for and provide counselling on menopause medicine regarding osteoporosis, cardiovascular disease, and breast cancer screening as major public health practices. To address this care discrepancy, one of our authors (WS) has developed the Johns Hopkins Menopause App, which is a point-of-care instrument to aid healthcare providers (MD, NP, PA) in counselling and treating menopausal women. This app hopes to improve knowledge and comfort of the providers and to increase patient satisfaction with menopause care. 


\section{Acknowledgements}

We would like to acknowledge AARP for their assistance with the administering of the survey and data analysis.

\section{Disclaimer}

The views expressed in the submitted article are our own and not an official position of the institution or funder.

\section{References}

[1] Bureau UC. Age and Sex Composition in the United States: 2010. https://www.census.gov/data/tables/2010/demo/ageand-sex/2010-age-sex-composition.html. Accessed September 7, 2018.

[2] Singh B, Liu XD, Der-Martirosian C, et al. Menopausal issues: A national probability sample of US women. Altern Ther Health Med. 2007; 13 (3): 24-29. http://www.ncbi.nlm.nih.gov/pubmed/17515021. Accessed May 19, 2018.

[3] Simon JA, Kokot-Kierepa M, Goldstein J, Nappi RE. Vaginal health in the United States. Menopause. 2013; 20 (10): 10431048. doi: 10.1097/GME.0b013e318287342d.

[4] Kingsberg SA, Wysocki S, Magnus L, Krychman ML. Vulvar and Vaginal Atrophy in Postmenopausal Women: Findings from the REVIVE (REal Women's VIews of Treatment Options for Menopausal Vaginal ChangEs) Survey. J Sex Med. 2013; 10 (7): 1790-1799. doi: 10.1111/jsm.12190.

[5] Kingsberg SA, Krychman M, Graham S, Bernick B, Mirkin S. The Women's EMPOWER Survey: Identifying Women's Perceptions on Vulvar and Vaginal Atrophy and Its Treatment. $J \quad S e x \quad M e d . \quad 2017 ; \quad 14$ (3): 413-424. doi: 10.1016/j.jsxm.2017.01.010.

[6] Cumming GP, Currie HD, Moncur R, Lee AJ. Web-based survey on the effect of menopause on women's libido in a computer-literate population. Menopause Int. 2009; 15 (1): 812. doi: $10.1258 / \mathrm{mi} .2009 .009001$.

[7] Manson JE, Chlebowski RT, Stefanick ML, et al. Menopausal hormone therapy and health outcomes during the intervention and extended poststopping phases of the Women's Health Initiative randomized trials. JAMA. 2013; 310 (13): 13531368. doi: 10.1001/jama.2013.278040.

[8] Anderson GL, Limacher M, Assaf AR, et al. Effects of conjugated equine estrogen in postmenopausal women with hysterectomy: the Women's Health Initiative randomized controlled trial. JAMA. 2004; 291 (14): 1701-1712. doi: 10.1001/jama.291.14.1701.

[9] Writing Group for the Women's Health Initiative Investigators WG for the WHI. Risks and Benefits of Estrogen Plus Progestin in Healthy Postmenopausal Women: Principal Results From the Women's Health Initiative Randomized Controlled Trial. JAMA J Am Med Assoc. 2002; 288 (3): 321333. doi: 10.1001/jama.288.3.321.

[10] Sprague BL, Trentham-Dietz A, Cronin KA. A sustained decline in postmenopausal hormone use: results from the National Health and Nutrition Examination Survey, 19992010. Obstet Gynecol. 2012; 120 (3): 595-603. doi: 10.1097/AOG.0b013e318265df42.

[11] Williams RE, Kalilani L, DiBenedetti DB, Zhou X, Fehnel SE, Clark R V. Healthcare seeking and treatment for menopausal symptoms in the United States. Maturitas. 2007; 58 (4): 348-358. doi: 10.1016/j.maturitas.2007.09.006.

[12] Rossouw JE, Prentice RL, Manson JE, et al. Postmenopausal Hormone Therapy and Risk of Cardiovascular Disease by Age and Years Since Menopause. JAMA. 2007; 297 (13): 14651477. doi: 10.1001/jama.297.13.1465.

[13] Stuenkel CA, Davis SR, Gompel A, et al. Treatment of Symptoms of the Menopause: An Endocrine Society Clinical Practice Guideline. J Clin Endocrinol Metab. 2015; 100 (11): 3975-4011. doi: 10.1210/jc.2015-2236.

[14] Salpeter SR, Walsh JME, Greyber E, Salpeter EE. Brief report: Coronary heart disease events associated with hormone therapy in younger and older women. A meta-analysis. $J$ Gen Intern Med. 2006; 21 (4): 363-366. doi: 10.1111/j.15251497.2006.00389.x.

[15] Boardman HMP, Hartley L, Eisinga A, et al. Hormone therapy for preventing cardiovascular disease in post-menopausal women. Cochrane database Syst Rev. 2015; (3): CD002229. doi: 10.1002/14651858.CD002229.pub4.

[16] Manson JE, Ames JM, Shapiro M, et al. Algorithm and mobile app for menopausal symptom management and hormonal/nonhormonal therapy decision making. Menopause. 2015; 22 (3): 247-253. doi: 10.1097/GME.0000000000000373.

[17] Christianson MS, Ducie JA, Altman K, Khafagy AM, Shen W. Menopause education. Menopause. 2013; 20 (11): 1120-1125. doi: 10.1097/GME.0b013e31828ced7f.

[18] Christianson MS, Washington CI, Stewart KI, Shen W. Effectiveness of a 2-year menopause medicine curriculum for obstetrics and gynecology residents. Menopause. 2016; 23 (3): 275-279. doi: 10.1097/GME.0000000000000531. 